\title{
The Prevalence of COVID-19 in Jizan Region-Saudi Arabia: A Demographic Analysis
}

\author{
Seham Sahli and Sharafaldeen Bin Nafisah
}

\begin{abstract}
BACKGROUND The unrelenting pandemic of the SARS CoV2 (COVID-19) pleads for re-examining predictors of infection and containment measures, once again. AIMS The researchers aim to investigate the prevalence of COVID-19 in Jizan region to analyse the demographic details of the population, to examine the quarantine predictors and the prescription of zinc and azithromycin. METHODS The researcher reviewed the Jizan region data obtained from the Ministry of Health of Saudi Arabia and performed a crosssectional study from September 1st, 2020 - September 29th, 2020. The researchers surveyed people from the same region to collect and analyse demographic and quarantine data. RESULTS The total number of positive cases was 11,752 patients in the Jizan region since the start of the pandemic. The prevalence of infection is $0.84 \%$ with a mortality rate of $1.73 \%(n=257)$. Out of 328 participants, $46.4 \%(n=148)$ acquired the infection with an admission rate of $1.6 \%(n=5)$. We noted two predictors for infection in the region: female gender and being married. Furthermore, males were more likely to be admitted than females and irrespective of age and chronic diseases. The quarantine after contact with a probable case or after travel showed an inverse relationship with the age; and in particular young females stratum, $p<0.05$. One third received zinc supplementation, whereas the majority $82.4 \%$ was not prescribed azithromycin. CONCLUSION Overall, the researchers provide a region-specific analysis that uncovers important infection determinants for COVID-19 infection, which should be taken into consideration when designing and implementing health promotions programs.
\end{abstract}

Index Terms - COVID-19, Jizan, Demographic Analysis

\section{INTRODUCTION}

SARS CoV2 (COVID-19) was initially reported in Wuhan, China, in December 2019. In a few months, the virus spread rapidly across the globe, and the World Health Organization (WHO) declared the outbreak to be a Public Health Emergency of international concern [1], [2]. In Saudi Arabia, however, the first case reported on March 2nd, 2020, and within the same month, the infection reported in different regions [3]. However, the lack of demographic analysis of people at risk makes public health efforts fruitless. The reason is, it does not address people's behaviour during the pandemic. Furthermore, an analysis of the demographic and the predicators for the dissemination of infection is imperative given the persistence of the pandemic despite several containment measures.

Jizan region is a coastal region in the south of Saudi Arabia along the Red Sea. It comprises of Jizan city and several associated provinces with a total population of approximately 1.4 million [4]. Nonetheless, the region lacks the demographic details of infected cases and those at risk. The researchers

Seham Sahli, Sharafaldeen Bin Nafisah are with Emergency DepartmentKing Fahd Medical City, Saudi Arabia, e-mail: Seham.sahli@hotmail.com, email: sbinnafisah@kfmc.med.sa

Seham Sahli is the corresponding author. therefore, aim to investigate the prevalence of COVID-19 in such region and to analyse the demographic details of the population concerning the age, gender, marital status, quarantine and the prescription of zinc and azithromycin. It also aims to infer on predictors for infection and quarantine, and finally, aims to conclude with several region-specific policies.

\section{Methods}

The researchers reviewed data obtained from the Ministry of Health of Saudi Arabia concerning COVID-19 in the Jizan region. The researchers also performed a cross-sectional study to survey people from the same region. The inclusion criteria were adults of both genders of more than 18 years of age who was in the region during the period of infection. The data collected through an Arabic structured questionnaire from the period of September 1st, 2020- September 29th, 2020, using an online survey tool. The researchers gathered the demographic details of the participants, factors associated with quarantine and whether they acquired the infection. It is also inquired by the researchers about the use of the commonly prescribed zinc supplements and azithromycin. The researchers included a photo of Azithromycin drug package for the ease of identification and the brand names of medications that is available in the region. The sample size is selected through online calculator www.calculator.net to calculate the sample population size; using a confidence level of $95 \%$ and a margin of error $5 \%$; the resulted sample size was 385 participants.

\section{Results}

\section{Demographic:}

The total population included in the analysis was 328 participants which entail $85.2 \%$ of the region calculated sample. The demographic details of the participants illustrated in Table 1.

Prevalence of infection:

The total number of positive cases was 11,752 patients in the region since the start of the pandemic and until October $5^{\text {th }}, 2020$. Region-specific mortality data revealed a mortality rate of $1.73 \%(\mathrm{n}=257)$ with a prevalence of infection of $0.84 \%$ of the region total population. However, the number of positive cases comprises $3.49 \%$ of the total cases in Saudi Arabia, knowing that the total number of cases reported in Saudi Arabia is 336,387 until October 5th, 2020 [5]. Table 2 illustrates the number of cases, recovery and mortality rates in the city and provinces of the Jizan region. Figure 1 also illustrates the number of cases in different parts of the Jizan region to the total number of populations. 


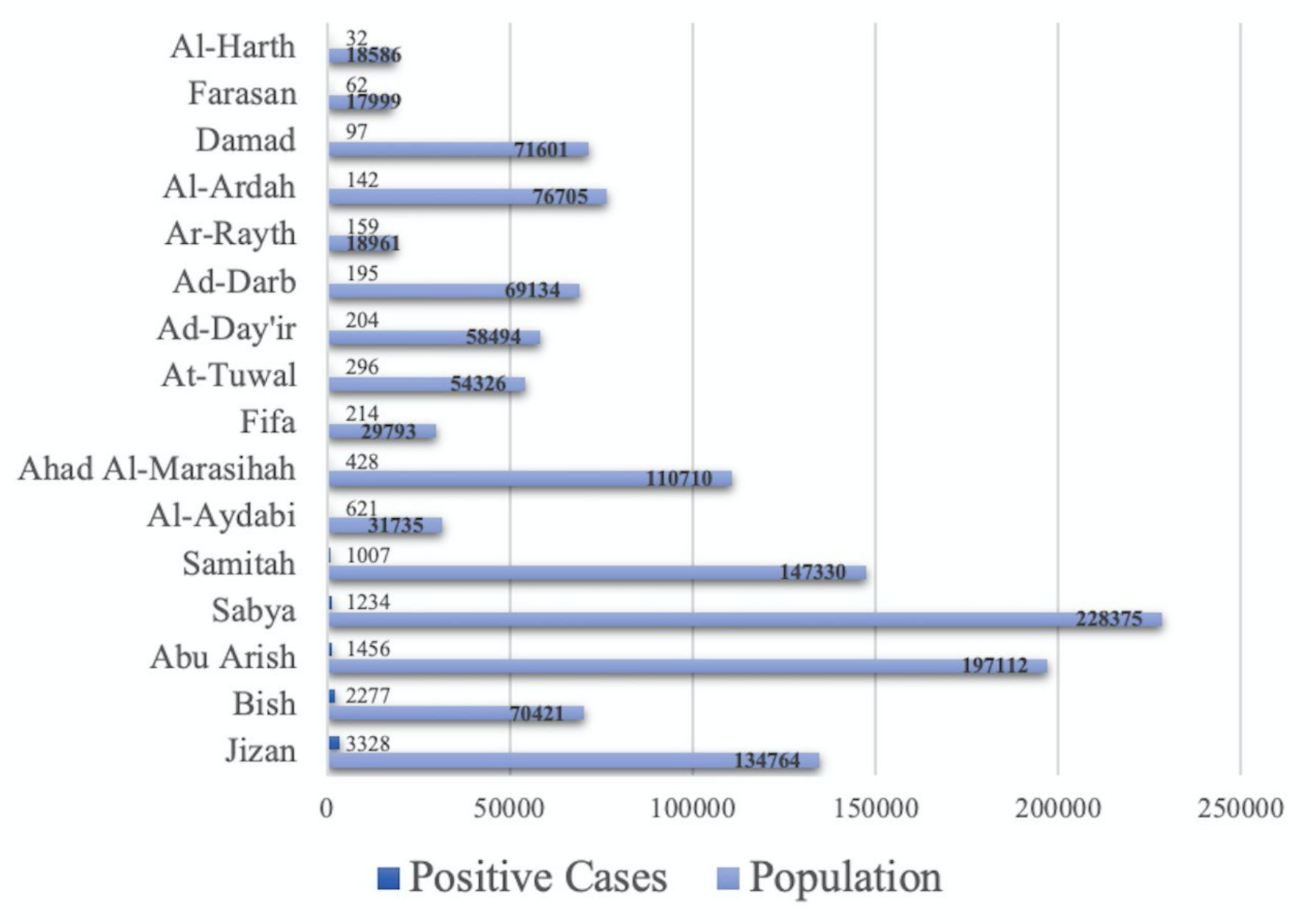

Fig. 1. The number of cases in Jizan city and its provinces in relation to the total number of populations.

In the sample, however, $46.4 \%$ ( $\mathrm{n}=148)$ acquired COVID-19 infection; while only $1.6 \%$ got admitted $(\mathrm{n}=5)$. Among those who got admitted $0.6 \%(\mathrm{n}=2)$ required oxygen during their illness.

Predictors for infection/complications:

The age of the participants was neither a predictor for developing the infection nor the need for admission, $\mathrm{p}>0.05$. Nevertheless, the number of females who acquire the infection outnumber males in $60.1 \%, 39.9 \%$, respectively-although such difference was not statistically significant to predict infection, $\mathrm{p}>0.05$. Males, on the other hand, were more likely to require admission than females, using Fisher's Exact Test, $p$ $<0.05$. Interestingly, the infection was more common among married participants $(65.5 \%)$ in comparison to single (32.4\%), widowed $(0.7 \%)$ and divorced $(1.4 \%),\left[\mathrm{X}^{2}(3)=8.33, \mathrm{p}<0.05\right]$. Besides, an underlying chronic disease did not predict the propensity to infection or the requirement for admission, $\mathrm{p}$ $>0.05$.

\section{Quarantine:}

$60.8 \%(\mathrm{n}=189)$ reported being quarantined after contacting with a probable case or after a history of travel. Likewise, $57.5 \%(n=153)$ reported been quarantined because of symptoms. However, quarantine after contact with a probable case or after travel showed an inverse relationship with the age, calculated using a Fisher's Exact Test $=15.6, \mathrm{p}<0.05$. Females were more likely to be quarantined for the same reasons, calculated using a Continuity Correction test $=13.42, \mathrm{p}<0.05$. Neither did the age of the participants, nor their gender predict the need for quarantine based on the symptoms, $p>0.05$.

Furthermore, patients with chronic diseases were less likely to be quarantined in comparison to medically-free patients, Fisher's Exact Test $=16.42, \mathrm{p}<0.05$. However, quarantine based on the manifestation of respiratory symptoms did not differ between those with chronic diseases and who were medically-free, $p>0.05$.

Zinc and azithromycin medications:

$34.5 \%(\mathrm{n}=51)$ of participants who acquired the infection prescribed zinc supplementation. The majority of participants of $82.4 \%$ reported not receiving azithromycin as part of their management plan $(n=122)$.

\section{Discussion}

To the time of this writing, this is the first article to analyse the demographic-details of COVID-19 in the region of Jizan. It is also noted that, although the prevalence of infection and the mortality rate is similar to other provinces [5], several critical 
demographic determinants emerge. For instance, males were more likely to require admission than females which raises a question about the negative attitude toward early medical attention. Furthermore, younger adult and in particular females were more likely to be quarantined secondary to contact with probable cases. It is prudent to assert and to infer on the need for health promotion strategies that promote social isolation among young females in such a province and to advocate against socialisation. On the other hand, there is a need to foster a concept of social isolation within the house, especially when there is a family member with respiratory symptoms. The lack of such isolation explains the high percentage of a married individual who acquired the infection, noted in the analysis.

Beyond the demographics, the researchers noted that patients with chronic diseases were less likely to be quarantined despite symptoms and despite contacting with probable cases. Given the propensity of the infection in such strata and the poor prognostic values, the researchers, therefore, encourage more adherences with the quarantine guideline issued by the Ministry of Health of Saudi Arabia [6] which take into consideration chronic diseases as a predictor for poor outcome. [7]

Furthermore, the researchers noted that a large segment did not use zinc supplement during their illness. Zinc supplementation showed antiviral properties in reducing the risk of acquiring the infection, the duration and severity of the COVID-19 illness [8], [9], [10]. Nevertheless, whether such usage needs emphasis; still more data for its benefit to be made first. Moreover, it is a common practice to prescribe azithromycin for outpatient management for the treatment of COVID-19 [11]. The rationale is because of its antiviral profile and immunomodulatory properties [12].

Nonetheless, the antibiotic failed to produce a benefit in clinical trials [13]. Subsequently, several guidelines-including local ones did not advocate azithromycin as a standard of care for outpatient management. Its usage recommended only for mechanically ventilated patients, and even then, it is a weak recommendation [14], [15]. Therefore, such an attitude of not dispensing empirically azithromycin should be maintained and strengthened. The limitation of this study lies in the lack of demographic details of other regions and provinces that can be used for comparison. The researchers advocate further studies that target the behaviour and attitude of the population through a region-specific analysis in order to understand the virus further and the venue in which the virus still resides.

\section{CONCLUSION}

Overall, the researchers provide a region-specific analysis that uncovers important infection determinants for COVID19 infection. Such demographic determinants are essential in shaping healthcare policies and procedures and should ameliorate health promotions programs. Understanding the cultural aspect-which differs between provinces within the same country, is crucial for the success of any containment efforts.

\section{REFERENCES}

[1] 2020. [Online]. Available: https://www.who.int/dg/speeches/detail/whodirector-general-s-opening-remarks-at-the-mission-briefing-on-covid19

[2] N. Zhu, D. Zhang, W. Wang, X. Li, B. Yang, J. Song, X. Zhao, B. Huang, W. Shi, R. Lu, P. Niu, F. Zhan, X. Ma, D. Wang, W. Xu, G. Wu, G. F. Gao, and W. Tan, China Novel Coronavirus Investigating and Research Team. A Novel Coronavirus from Patients with Pneumonia in China, vol. 382, pp. 727-733, 2019.

[3] Ministry and Health, 2020. [Online]. Available: https://www.moh.gov. $\mathrm{sa} / \mathrm{en} / \mathrm{Ministry} / \mathrm{MediaCenter/News/Pages/News-2020-03-02-002.aspx}$

[4] 2020. [Online]. Available: https://www.stats.gov.sa/sites/default/files/ jazan_region_ar.pdf

[5] [Online]. Available: https://covid19.cdc.gov.sa/daily-updates/

[6] [Online]. Available: https:/www.moh.gov.sa/CCC/healthp/regulations/ Documents/Coronavirus\%20Disease\%202019\%20Guidelines\%20v1.1. .pdf

[7] H. Liu, S. Chen, M. Liu, H. Nie, and H. Lu, "Comorbid Chronic Diseases are Strongly Correlated with Disease Severity among COVID19 Patients: A Systematic Review and Meta-Analysis," Aging and disease, vol. 11, no. 3, pp. 668-668, 2020. [Online]. Available: 10.14336/ad.2020.0502;https://dx.doi.org/10.14336/ad.2020.0502

[8] I. Wessels, B. Rolles, and L. Rink, "The Potential Impact of Zinc Supplementation on COVID-19 Pathogenesis," Frontiers in Immunology, vol. 11, 2020. [Online]. Available: 10.3389/fimmu.2020.01712; https: //dx.doi.org/10.3389/fimmu.2020.01712

[9] S. Arentz, G. Yang, J. Goldenberg, J. Beardsley, S. P. Myers, D. Mertz, S. Leeder, and J. Hunter, "Clinical significance summary: Preliminary results of a rapid review of zinc for the prevention and treatment of SARS-CoV-2 and other acute viral respiratory infections," Advances in integrative medicine, 2020.

[10] A. Kumar, Y. Kubota, M. Chernov, and H. Kasuya, "Potential role of zinc supplementation in prophylaxis and treatment of COVID19," Medical Hypotheses, vol. 144, pp. 109 848-109 848, 2020. [Online]. Available: 10.1016/j.mehy.2020.109848;https://dx.doi.org/10. 1016/j.mehy.2020.109848

[11] C. E. Oldenburg and T. Doan, "Azithromycin for severe COVID-19," The Lancet, vol. 396, no. 10256, pp. 936-937, 2020. [Online]. Available: 10.1016/s0140-6736(20)31863-8;https://dx.doi.org/10.1016/ s0140-6736(20)31863-8

[12] N. Bleyzac, S. Goutelle, L. Bourguignon, and M. Tod, "Azithromycin for COVID-19: More Than Just an Antimicrobial?" Clinical Drug Investigation, vol. 40, no. 8, pp. 683686, 2020. [Online]. Available: 10.1007/s40261-020-00933-3;https: //dx.doi.org/10.1007/s40261-020-00933-3

[13] R. Furtado, O. Berwanger, and H. A. Fonseca, "Azithromycin in addition to standard of care versus standard of care alone in the treatment of patients admitted to the hospital with severe COVID-19 in Brazil (COALITION II): a randomised clinical trial," Lancet, vol. 396, pp. $31862-31868,2020$

[14] W. Alhazzani, F. Al-Suwaidan, Z. A. Aseri, A. A. Mutair,

G. Alghamdi, A. Rabaan, M. Algamdi, A. Alohali, A. Asiri, M. Alshahrani, M. Al-Subaie, T. Alayed, H. Bafaqih, S. Alkoraisi, S. Alharthi, F. Alenezi, A. A. Gahtani, A. Amr, A. Shamsan, Z. A. Duhailib, and A. Al-Omari, "The saudi critical care society clinical practice guidelines on the management of COVID-19 patients in the intensive care unit," pp. 27-27, 2020. [Online]. Available: 10.4103/sccj.sccj 15 20;https://dx.doi.org/10.4103/sccj.sccj 1520

[15] W. Alhazzani, M. $\bar{H}$. Møller, and Y. M. Arabi, "Surviving Sepsis Campaign: Guidelines on the Management of Critically Ill Adults with Coronavirus Disease 2019 (COVID-19)," Crit Care Med, vol. 48, pp. 440-469, 2020 . 
TABLE I

THE DEMOGRAPHIC DETAILS OF THE SURVEYED PARTICIPANTS.

\begin{tabular}{|c|c|c|}
\hline Characteristic & $n$ & Valid \% \\
\hline \multicolumn{3}{|l|}{ Age groups } \\
\hline $18-30$ & 194 & 59.3 \\
\hline $30-40$ & 97 & 29.7 \\
\hline $40-50$ & 31 & 9.5 \\
\hline$>50$ & 5 & 1.5 \\
\hline \multicolumn{3}{|l|}{ Gender } \\
\hline Female & 198 & 60.6 \\
\hline Male & 129 & 39.4 \\
\hline \multicolumn{3}{|l|}{ Marital status } \\
\hline Single & 120 & 37.0 \\
\hline Married & 191 & 59.0 \\
\hline Widowed & 1 & 0.3 \\
\hline Divorced & 12 & 3.7 \\
\hline \multicolumn{3}{|l|}{ Chronic disease } \\
\hline Yes (unspecified) & 46 & 14.2 \\
\hline No & 253 & 78.1 \\
\hline Diabetic mellitus & 8 & 2.5 \\
\hline Hypercholesterolemia & 2 & 0.6 \\
\hline Hypertension & 10 & 3.1 \\
\hline Diabetic Mellitus \& Hypercholesterolemia & 1 & 0.3 \\
\hline Diabetic Mellitus \& Hypertension & 4 & 1.2 \\
\hline
\end{tabular}

TABLE II

THE NUMBER OF CASES, RECOVERY AND MORTALITY RATE IN THE CITY AND DIFFERENT PROVINCES OF THE JIZAN REGION

\begin{tabular}{llllll}
\hline Provinces & Cases $(\mathrm{n})$ & Recovery $(\mathrm{n})$ & Recovery $(\%)$ & Mortality $(\mathrm{n})$ & Mortality rate (\%) \\
Jizan & 3,328 & 2,823 & 84.82 & 109 & 3.27 \\
Bish & 2,277 & 2,180 & 95.74 & 24 & 1.05 \\
Abu Arish & 1,456 & 1,290 & 88.59 & 32 & 2.19 \\
Sabya & 1,234 & 1,116 & 90.44 & 45 & 3.64 \\
Samitah & 1,007 & 928 & 92.15 & 19 & 1.88 \\
Al-Aydabi & 621 & 577 & 92.91 & 0 & 0 \\
Ahad Al-Marasihah & 428 & 366 & 85.51 & 5 & 1.16 \\
Fifa & 214 & 192 & 89.72 & 0 & 0 \\
At-Tuwal & 296 & 171 & 89.90 & 2 & 0.96 \\
Ad-Day'ir & 204 & 193 & 94.61 & 3 & 1.47 \\
Ad-Darb & 195 & 175 & 89.74 & 6 & 3.07 \\
Ar-Rayth & 159 & 131 & 82.39 & 0 & 0 \\
Al-Ardah & 142 & 128 & 90.14 & 7 & 4.93 \\
Damad & 97 & 84 & 86.59 & 4 & 4.12 \\
Farasan & 62 & 59 & 95.16 & 1 & 1.61 \\
Al-Harth & 32 & 28 & 87.5 & 0 & 0 \\
Total & 11,752 & 10,541 & 90.08 & 257 & 1.73 \\
\hline
\end{tabular}

